\title{
Über die geographische Bedeutung des Grundeigentums
}

Unter dem Eindruck der gegenwärtigen Veränderung unserer lebensräumlichen Situation machen sich in der Geographie zunehmend Tendenzen bemerkbar, den räumlich differenzierten Wohlstand bzw. die allgemeine Lebensqualität der Menschheit als generelle Zielsetzung der geographischen, insbesondere der humangeographischen Arbeit zu erklären. So sind der globale Aspekt und jener des verantwortlichen Bewußtseins gegenüber der heutigen Dynamik für einige moderne Werke der Geographie bzw. der Umweltforschung bestimmend geworden, wie dies beispielsweise das Sammelwerk von THOMAS "Man's role in changing the face of the earth" oder neuestens auch die "WohlfahrtsGeographie» von D.M.SMITH erkennen lassen. Mögen sich die Beweggründe zur Erforschung des Lebensraumes Erde im einzelnen als individuellvielfältig erweisen: die globale Gegenwarts-Problematik erfaßt jeden Denkenden und fordert auch vom Geographen immer wieder eine kritische Neubesinnung.

Aus dieser Situation heraus sind die nachstehenden Ausführungen $\mathrm{zu}$ verstehen, vor allem als Folge von mehrjähriger Beobachtung aktueller Landschaftsveränderungen. Dabei hat sich u.a. die methodische Einsicht neu bestätigt, wonach das Grundeigentum im räumlichen Geschehen eine derartige Stellung einnimmt, daß es noch eine vermehrte systematische Würdigung durch die Geographie verdiente. Mit der Diskussion der verschiedenen räumlichen Aspekte des Grundeigentums sollte nicht nur ein allgemeines Verständnis für die Funktion des Eigentums im Landschaftsganzen geweckt, sondern, was für die angewandte Geographie und die Raumplanung wesentlich erscheint, konkrete Einsichten in das System der räumlichen Verfügungsgewalt eröffnet werden.

Es sei im folgenden versucht, die Eigentumsproblematik in ihrer wissenschaftsgeschichtlichen Position und in ihren Auswirkungen auf den geographischen Raum zu verdeutlichen. Gleichsam als Ausgangspunkt ließe sich zuerst eine breit juristisch angelegte Begriffsklärung denken, welche den Terminus "Grundbesitz», wie er in geographischen Arbeiten häufig verwendet wird, in seiner Bezüglichkeit zum Eigentumsbegriff klären würde. Diese Aufgabe muß jedoch dem Juristen überlassen blei- ben; wir beschränken uns lediglich auf die grundsätzliche Feststellung, daß «Bodeneigentum» dem «Bodenbesitz» insofern übergeordnet ist, als «Eigentum» das formale Besitzrecht an einer Sache begründet, wobei der Eigentümer im Rahmen der Rechtsordnung über «eine vielseitige, weitreichende, ja die allerumfassendste rechtliche Herrschaft, die an einer Sache überhaupt möglich ist» verfügt (TUOR-SCHNYDER 1969, S. 480). Dagegen umschreibt "Besitz» das konkrete Nutzungsrecht an einer Sache, wie es das Schweizerische Zivilgesetzbuch (Art.9191) definiert: "Wer die tatsächliche Gewalt über eine Sache hat, ist ihr Besitzer». Besitz ist direkt mit der Nutzungsweise eines Gutes verknüpft und zwar in selbständiger oder unselbständiger Form. "Selbständiger Besitzer ist zunächst jener, der die Sache als Eigentümer in der Gewalt hat, unselbständiger Besitzer jener, dem der Eigentümer die Sache zu einem dringlichen oder persönlichen Recht übertragen hat, der also seinen Besitz von jenem ableitet: der Nutznießer, Mieter, Pächter...” (TUOR-SCHNYDER 1969, S. 435). Da sich unsere Überlegungen vor allem am räumlichgenerellen Zusammenhang zwischen Boden und Mensch orientiert, sind wir primär eher an der vollen Verfügungsgewalt über Grund und Boden interessiert, weshalb wir - mit ähnlicher Fragestellung wie R. JOLLER - dem Begriff des "Grundeigentums» ein etwas stärkeres Gewicht beimessen. Gleichwohl soll in unseren Erörterungen "Grundeigentum» in einem sprachlich allgemeinen, juristisch noch unentschiedenen Sinne verwendet werden; er könnte auch "Grundbesitz» mitbeinhalten. Wesentlicher räumlicher Ausdruck der Eigentumsverhältnisse ist das «Grundstück» (auch "Liegenschaft» oder «Parzelle»). Darunter verstehen wir jede «zusammenhängende Bodenfläche mit genügend bestimmten Grenzen, die im Grundbuch bzw. Liegenschafts- und Servitutenregister auf einem eigenen Blatt individualisiert ist» (JOLLER 1969, S. 22). Somit wird das Grundbuch zu einer methodisch wichtigen Verbindung im Untersuchungsfeld Mensch Raum - Bodennutzung.

Prof. Dr. W. A. Gallusser, Ordinariat für Humangeographie, Geographisches Institut der Universität Basel,

Klingelbergstraße 16, 4056 Basel. 


\section{Grundeigentum als Funktion der Kultur}

Fragt man nach dem Zusammenhang zwischen dem Grundeigentum und dem geographischen Raum, so ist aufs erste auf die Tatsache hinzuweisen, daß sich Landschaft primär aus der Gesamtheit der aus Grundeigentum abgeleiteten "Grundstücke» aufbaut; hinter dem also konstruierten, sinnhaften Raum ersteht ein zugeordneter, nur über das Grundbuch erfaßbarer «juristischer Raum». Als Funktionalraum ist er unsichtbar und blieb damit dem geographischen Zugriff entrückt. Diese Tatsache steht m.E. in Verbindung zur relativ verspäteten Entfaltung des funktionalen Denkens in der Geographie, wobei sich wissenschaftsgeschichtlich zwar schon recht früh erste Darlegungen unserer Problematik feststellen lassen, allerdings im noch recht allgemeinen Zusammenhang des Grundeigentums als Funktion der Kultur. In dieser Bedeutung werden etwa in der klassischen Siedlungsgeographie von G.SCHWARZ die Aspekte des Grundeigentums berücksichtigt, hat doch die Autorin versucht, die Gestaltung der Wohnplätze u.a. auf grundherrschaftliche Bedingungen zurückzuführen. Sie betrachtet die Grundherrschaft als einen bestimmten Ausdruck der Sozialverhältnisse, welche dem zeitlichen Wandel unterliegen und eine besitzmäßige Differenzierung des Raumes erbringen. Insbesondere würden danach die Erbsitten und die staatlichen Eingriffe in die grundherrschaftlichen Zustände wesentliche Veränderungen des Siedlungsraumes bewirken. Tatsächlich unterscheiden sich die Anerben- und die Realteilungsgebiete nicht nur eigentumsrechtlich, sondern in ihrer ganzen Raumstruktur und Physiognomie, und die Länder Europas, in denen die grundherrschaftlichen Bindungen während des letzten Jahrhunderts weitgehend aufgehoben worden sind, verzeichnen eine vielfältig individuelle Entwicklung von Bodennutzung und Siedlung, wodurch das heutige Landschaftsbild entscheidend mitgeprägt worden ist. In dieser methodischen Sicht widerspiegelt sich die Orientierung der europäischen Geographie: ausgehend von einem Vorverständnis für die feudalzeitlichen Wurzeln unserer europäischen Kulturlandschaft, wurden Einsichten in das durch die Europäisierung der Erde ausgeweitete Forschungsfeld entwickelt. Die Unübertragbarkeit europäischer Sachverhalte auf die exotischen "Fremdstrukturen» hemmte die Ausbildung einer auch die Eigentumsverhältnisse voll umfassenden geographischen Methodik, so daß sich vornehmlich der großräumige Vergleich und die aus historisch-differenzierendem Bemühen entstammende «KulturkreisGeographie» entfaltete. Eine verfeinerte Weiterentwicklung dieser vergleichenden Kulturkreisforschung sehe ich in H. BOBEKs Werk, vor allem in seinem klassischen Aufsatz über «die Hauptstufen der
Gesellschafts- und Wirtschaftsentfaltung» (1959), worin er die kulturräumliche Bedeutung der Grundherrschaft auch sozialgeographisch klar herausgearbeitet hat. Daß die Eigentumsproblematik im kulturgeographischen Schrifttum erst relativ spät zum Tragen gekommen ist, belegen die Beiträge von L. WAIBEL 1928 und H. HASSINGER 1929; so wird die Frage des Grundeigentums in WAIBELs Abhandlung über die Sierra Madre de Chiapas nur indirekt über die verschiedenen Betriebsformen angetönt und HASSINGER sieht zwar in seinem methodischen Aufsatz durchaus Grund und Boden als «Kapitalträger», aber nur in dieser eingeschränkten wirtschaftlichen Bedeutung. Offenbar liegt hier die ganze Problematik der räumlichen Besitzordnung noch im Gesamtkomplex der Kulturlandschaft eingebunden, gedanklich zuweilen wohl etwas deutlicher als Funktion der jeweiligen gesellschaftlichen und kulturellen Situation erkannt, wie das in P. H.SCHMIDTs Wirtschaftsgeographie schon 1932 innerhalb eines speziellen Kapitels über den Bodenbesitz ausgesprochen wird: "So steht der Besitzstand des Bodens in jedem Lande unter dem Einfluß von Bodenart und Klima. Aber diese sind keineswegs alleinbestimmend. Die Entscheidung hängt $a b$ von der sozialen Entwicklung des Volkes. Auch wenn nicht mehr, wie unter feudalen Verhältnissen, der Grundbesitz allein Macht und Geltung beansprucht, so sehen wir doch auch heute überall die sozialen Entwicklungsstufen, die in so mannigfachen Formen auf der Erde sich entfaltet haben, im engsten Zusammenhang mit der Verteilung von Grund und Boden stehen... wenn wir die Grundbesitzverhältnisse eines Landes erfahren, eröffnet sich uns ein Bild einer sozialen Entwicklungsstufe; es erschließen sich uns die Machtverhältnisse der sozialen Schichten im Staate, die Wirtschaftskraft des nationalen Bodens; wir sehen, wie Freiheit und Dienstbarkeit, Wohlstand und Armut von der Bodenverteilung abhängen...(a.a.O., S. 283 f.).

Seither sind auf soziologischer, rechtshistorischer und ethnologischer Seite detaillierte Studien über die soziokulturellen Hintergründe der Bodenverteilung in der Dritten Welt bekannt geworden. Fortschritte, die sich auf die Zielsetzung einer ebenso weiterentwickelten Geographie anregend auswirken dürften. Die genaue Kenntnis dieser unsichtbaren, aber bestimmenden Grundkonturen der Kulturlandschaft bietet eine hilfreiche Grundlage, um die unausweichliche Anpassung der Lebensverhältnisse vieler Länder aus den Gegebenheiten von gestern an jene von heute und morgen vornehmen zu können. Ein breites Schrifttum, auch von geographischen Autoren, hat sich dieser dynamischen $\mathrm{Zu}-$ sammenhänge - vor allem der Landreform in der Dritten Welt - angenommen. Als Beispiel sei etwa die Studie von R. CRESSWELL 1970 angeführt, worin die Grundstruktur der maronitischen Kulturland- 
schaft von Boqsmaya (Nordlibanon) hinsichtlich der naturräumlichen Disposition, des Grundbesitzes und der Bodennutzungsverhältnisse beschrieben wird. Diese sorgfältig belegte Analyse klärt den Zusammenhang zwischen dem Verwandtschaftssystem und der räumlichen Ordnung des Eigentums, wodurch die kulturräumliche Eigenart und Problematik des Untersuchungsraumes wesentlich erhellt werden.

In ähnlicher Ausrichtung sind ebenso die touristische Erschließung und die Verstädterung im Spiegel des Grundeigentums von Geographen untersucht worden (z. B. durch ERIKSEN, RUPPERT, VORLAUFER), und zwar in einer derartigen methodischen Vielfalt, daß sich für den Verfasser eine gesonderte Darstellung der raumdynamischen Problematik des Grundeigentums aufgedrängt hat (GALLUSSER 1979).

\section{Grundeigentum und Bodennutzung}

Die Bodennutzung spielt in der geographischen Arbeit seit je eine zentrale Rolle, was für die Eigentumsverhältnisse noch nicht gilt. Da wir jedoch den geographischen Gehalt des Grundeigentums klären wollen, sei versucht, den Zusammenhang zwischen Grundeigentum und Bodennutzung grundsätzlich und im Blick auf die moderne Literatur zu skizzieren.

Der mit dem Eigentumsbegriff unlösbar verbundene Nutzungsaspekt darf in der geographischen Praxis als bekannt und methodisch einigermaßen als erprobt gelten. Hiezu hat vor allem die Sozialgeographie wesentlich beigetragen; nicht zuletzt belebte die «Sozialbrache»-Forschung diese Problematik, indem vor allem w. HARTKE 1956 das Nutzungsphänomen «Brachfallen» primär auf die direkten "Reaktoren», die Landbesitzer und danach auch auf die «externe» sozialökonomische Auslösersituation zurückgeführt hat. Wohl wissend, daß die eigentliche Verursachung bedeutender landschaftlicher Erscheinungen in den Außenbedingungen der gesamtökonomischen und gesamtgesellschaftlichen Entwicklung zu suchen sind, möchten wir - als bewusste Einschränkung - diese Außenbedingungen als gegeben hinnehmen und von unserer gezielten Betrachtung ausschließen. So ergibt sich als Kern der geographischen Problematik die Analyse von "Reaktionsketten» zwischen dem Menschen als "Agent modificateur» und seinem zugeordneten Raum. Die Münchner Schule hat nun mehrfach nachgewiesen, daß einer aktuellen räumlichen Erscheinung (z. B. Sozialbrache, Vergrünlandung, Aufforstung) eindeutige Verursachersituationen zugeordnet werden können, d.h. daß eine bestimmte Landnutzungssituation nur durch die analytische Rückführung auf den direkt für den jeweiligen Raumausschnitt verantwortlichen Menschen erklärt werden kann. Das heißt mit anderen Worten: erst die humangeographische Analyse der raumwirksamen Personen und Gruppen wird zu schlüssigen Erkenntnissen über die Raumnutzung führen. In diesem Kontext läßt sich nun die Berücksichtigung der Eigentumsverhältnisse geographisch rechtfertigen, indem die genaue Kenntnis der Besitzsituation die zugeordnete Bodennutzung nicht nur deutet, sondern ursächlich erklärt, oder wie eingangs festgehalten wurde -: das unsichtbare Ordnungsnetz des Grundeigentums bewirkt das sichtbare Gefüge des Nutzungsraums. Zahlreiche geographische Feldarbeiten, welche die aufwendige Grundbuchauswertung nicht umgangen haben, belegen diese direkte Aussagefähigkeit der Eigentumsverhältnisse bezüglich der Raumnutzung.

Im Zusammenhang mit der Verstädterung, d.h. mit der räumlichen Konzentration vielfältiger Nutzungsformen, wäre auf die zunehmende Differenzierung der Eigentumsverhältnisse hinzuweisen. Nicht nur für die Beurteilung stadträumlicher Erscheinungen, sondern immer, wenn der Geograph die Frage des Grundeigentums in seine Betrachtung einbezieht, sollte dies zunehmend in möglichst differenzierter Form geschehen. Vor allem dürfte es kaum mehr genügen, die Kategorie "Grundbesitz» unbesehen ihrer juristischen Bestimmung zu verwenden. Wie schon erwähnt, müßte abgeklärt werden, ob im jeweiligen, konkreten Fall Eigentum und Besitz als getrennte oder als einheitliche Qualität auftritt. Je nachdem wird die Reaktionskette zur Bodennutzung hin anders verlaufen; so unterscheidet sich Eigentümerverhalten i.d. R. vom Pächterverhalten, wobei sich zusätzlich noch regionale Unterschiede ergeben können, je nachdem ob ländliche, rand- oder kernstädtische Verhältnisse vorliegen. Nachdem sich in der Humangeographie die Berücksichtigung sozialer Kategorien einigermaßen durchgesetzt hat, würde nun die räumliche Eigentumsproblematik aus methodischen Gründen die juristische Vertiefung erfordern. Gerade für den Geographen, der in verschiedenen Erdräumen forscht, 'bedeutet diese juristische Vertiefung eine zusätzliche Mühe, denn die kulturgebundene Rechtsordnung läßt sich nicht ohne Verfälschung auf andere kulturräumliche Situationen übertragen. Grundbesitzgliederungen nach sozio-ökonomischen oder gesellschaftlich-kulturellen Kriterien, bzw. nach dem Wohnort werden in geographischen Fragestellungen relativ häufig angewendet, nicht immer jedoch mit expliziter Begründung. Als Beispiel für eine systematisch differenzierte Betrachtung von Eigentümerkategorien sei auf den Aufsatz von TH. LUNDEN 1977 über Stockholm hingewiesen. Er macht bewußt, daß die Landeigentümer eines bestimmten Territoriums je nach der Geometrie ihres Besitzes (Größe und Verteilung) und nach dem 
eigentumsrechtlichen Status (z. B. private Einzelbesitzer; Gemeinden, Staat; Privatgesellschaften) unterschiedliche Nutzungsinteressen im gleichen Raum (und zur selben Zeit) verwirklichen wollen; die Unvereinbarkeit ergibt sich einmal aus dem vorgegebenen Eigentümergefüge, das je nach seiner Komposition Nutzungskonflikte verschiedenen Ausmaßes hervorrufen kann, und aus der Tatsache, daß die Eigentümer als Raumnachbarn ihre Nutzungsabsichten verwirklichen (wollen). Die systematische Abklärung dieser raumdifferenten Eigentümerhierarchie wäre ein wertvoller Beitrag der Geographie besonders zur räumlichen Harmonisierung unterschiedlicher Nutzungsansprüche, wie dies LUNDEN anhand von regionalen Fallstudien überzeugend dargelegt hat.

\section{Grundeigentum als Ausdruck der Entwicklung}

Immer ist Grundeigentum, weil es den Boden mit einem für dieses Gut auch in der Zeit Verantwortlichen bezeichnet, durch einen «prospektiven $\mathrm{Ge}$ halt» gekennzeichnet. Auch diese Komponente wurde in einigen agrargeographischen Abhandlungen ansatzweise herausgearbeitet, indem etwa ein zunehmendes Pachtlandangebot als Vorstufe zur Eigentumsmobilität mit nachherigem Landnutzungswandel interpretiert worden ist. Gesamthaft betrachtet, wird aber die prospektive Aussagemöglichkeit der Eigentumsverhältnisse, die von raumplanerischem Interesse sein kann, durch uns Geographen noch zu wenig angewendet, vielleicht mit Ausnahme der planungsbezogenen Forschung in Mitteleuropa, Kanada und in Skandinavien. Die norwegische Geographin A.TSCHUDI behandelte 1968 die Landnutzungsproblematik im Naherholungsraum nördlich von Oslo als Teilaspekt verschiedener Eigentümerstrategien, d.h. mit einem Ansatz, der auch in LUNDENs Stockholmarbeit zum Ausdruck kam. Neben der Eigentumsverteilung wirkt sich auch die Eigentumsgröße maßgeblich auf die Nutzungs- und Planungsverhältnisse aus, indem der bäuerliche Kleinbesitz die angestammte Agrarnutzung gegenüber der Urbanisierung kaum mehr aufrechterhalten kann; dagegen ist eine aktive Dynamik einiger Großeigentümer (wie z. B. der Stadt Oslo) festzustellen, die das Gebiet im Zeichen der Forstwirtschaft und der städtischen Bedürfnisse zu beherrschen beginnen, gleichzeitig aber wegen der überschaubaren Eigentumsverhältnisse regionalplanerische Lösungen erleichtern. Die kanadische Regionalstudie von L.F. BRADLEY und A. BEAULIEU 1973 über die Prinz Edward-Insel entstammt, wie andere Regionalanalysen im Rahmen des «Canada Land Inventory», einem 1963 lancierten Programm zur Beschaffung von Grundlagen und
Richtlinien für die Regionalentwicklung im besiedelten Teil Kanadas. Ein wesentlicher Teil der Arbeit ist der Bodeneigentümeranalyse gewidmet, mit der knappen Begründung: "the characteristics, attitudes and intentions of the land-owners are at least as important as the physical character of the land itself. The pattern of landownership in a spatial context is also of major significance in land use planning» (a.a.O., S. 7). Weil der Eigentümer als Schlüsselposition im Landnutzungsgeschehen erkannt ist, wird er folgerichtig in der ganzen Vielfalt seiner besitzräumlichen Bindung erfaßt (Abb.1), um daraus auf Grund von eingehenden Befragungen planerische Folgerungen z. B. über den künftigen Landbedarf zu ziehen.

Eine weitere prospektive Aussage ergibt sich aus der systematischen Abklärung von Eigentums- und (damit meist verhängt) von Nutzungswechseln in der Landschaft, d.h. von "dynamischen Sequenzen» bestimmter Grundstücke. So zeigt Abb. 2 die allmähliche Veräußerung von Grundeigentum einer Bürgergemeinde im Berner Jura. Aus der Lage, der Größe und dem Zeitpunkt des Verkaufes läßt sich die Nutzungsabsicht des Käufers wie die Landpolitik des Verkäufers direkt belegen; bei Kenntnis der raumplanerischen Randbedingungen erleichtert die kartographisch fixierte Parzellen-Sukzession überdies eine Extrapolation der beobachteten Grundstücksbewegung in die nähere Zukunft.

$\mathrm{Da}$ Grundeigentum nach klar geordneten Abläufen «entwickelt» werden kann, dokumentieren CADMAN und AUSTIN-CROWE mit ihrem Lehrbuch über «Property development». Die Kenntnis des gezielten Entwicklungsprozesses, um Grundstücke für neue Nutzungen vorzubereiten, ermöglicht ebenfalls vorausschauende Aussagen über das Grundeigentum eines bestimmten Gebietes. Ist der Geograph mit den verschiedenen ineinander greifenden Phasen der «Eigentumsentwicklung» vertraut, d.h. zum Beispiel mit der Sequenz Bewertung, Vorbereitung, Ausführung und Verkauf, so vermag er innerhalb des vorgegebenen Gesetzesrahmens eines Staates oder einer Region örtliche Entwicklungsvarianten des Grundeigentums zu beurteilen. ${ }^{1)}$

1) Vgl. dazu den methodischen Beitrag des Verfassers am IGUSymposium "Cartographie de l'environnement et sa dynamique» in Caen 1979 (im Druck). 




Abb. 1:

\section{Sozialökonomische Gruppierung der Grundeigentümer}

O'Leary Area (Prince Edward-Insel, Canada).

Mit Angabe der absoluten Zahl der Eigentümer und des dazugehörigen Kulturlandes (nach BRADLEY und BEAULIEU, 1973). 


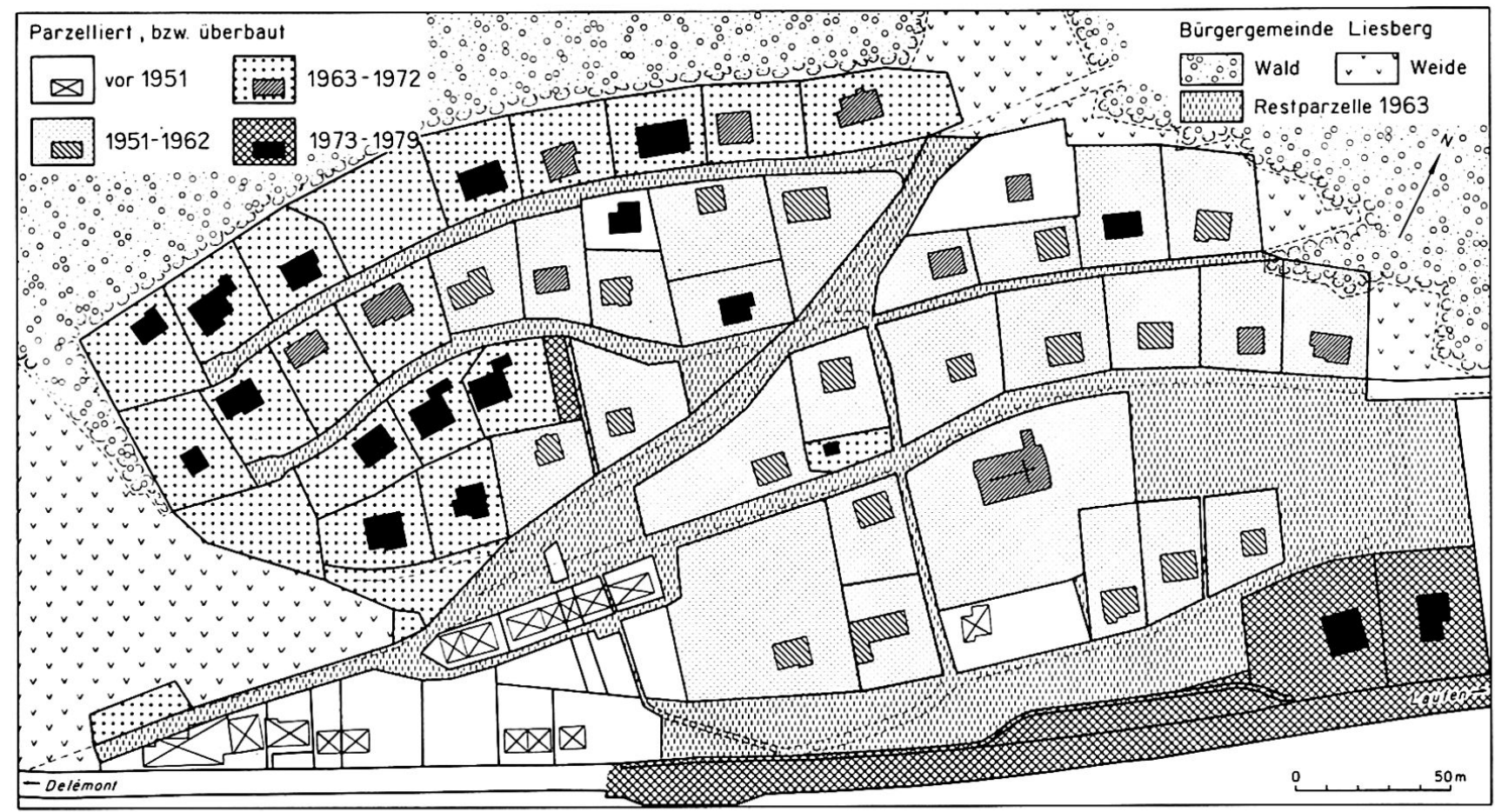

Abb. 2:

Aufteilung des Geländes Oberrütti der Bürgergemeinde Liesberg, 1951-79 (Plangrundlagen: Bruno Baur, Basel).

\section{Das Grundeigentum im räumlichen System}

Im Sinne des modernen Verflechtungsdenkens, das zunehmend auch für das "räumliche System» dienstbar gemacht wird, läßt sich grundsätzlich nach der Stellung und Funktion des Grundeigen-, tums im räumlichen System fragen. Weil mit dem Grundeigentum die legitime Macht des im Raum Handelnden, den Boden als Landschaftssubstanz Nutzenden, anvisiert ist, wäre ein Beitrag zur räumlichen Theorie des Grundeigentums von grundlegender Bedeutung, auch für eine Theorie der landschaftsgestaltenden Kräfte, d.h. für die Humangeographie schlechthin. Unsere vorläufigen Überlegungen sind als eine Aufmunterung zur wissenschaftlichen Diskussion und zur schrittweisen Erforschung der «Eigentumslandschaft» gedacht, u.z. in methodischer, nutzungsrechtlicher und in allgemeingeographischer Hinsicht.

Ein besonderes Augenmerk verdienen all jene geographischen Bemühungen, die sich bei der Modellierung nutzungsräumlicher Systeme explizit auch mit der Funktion des Landeigentümers befaßt haben. Hiezu gehört die Studie von P.CORDEY 1978. Wie sein Modell erkennen läßt, erscheint der Landeigentümer als Teil des raumbestimmenden Systems der Stadt, allerdings unter der marxistischen $\mathrm{Hy}$ pothese, wonach der Landeigentümer vor allem darauf bedacht sei, aus seinem Land eine maximale Bodenrente herauszuwirtschaften. Dadurch wird die Stellung des Grundeigners neben jener des Kapitalbesitzers und des Staates relativiert.

So bemerkenswert die Modellvorstellungen CORDEYs als Diskussionsbeitrag auch sind, so eindeutig sind sie von einer Wirtschaftstheorie abgeleitet und behaupten unausgesprochen die Dominanz des Ökonomischen über das Recht und den Raum. Liegt hierin nicht ein implizites Hinnehmen einer für Europa vielleicht realistischen Grundhaltung des Menschen zum Boden? Nicht, daß räumliche Erscheinungen marxistisch oder nichtmarxistisch erklärt werden, sondern, daß sie a priori nur wirtschaftlich gesehen und gedeutet werden, bedarf vorerst der geographischen Vertiefung und Ergänzung, u.z. im Blick auf die weltweite Raumwirklichkeit. Dabei ließe sich - wenn man A. WILDERMUTH 1970 in seiner Marx-Interpretation folgt feststellen, daß in der Diskussion des Grundeigentums bei Marx ein geophilosophischer Erdbezug deutlich wird: "Der Gedanke der leiblich integrierten Erde in die Tätigkeit des Menschen ist dazu unabdingbar. Denn Eigentum weist grundsätzlich zurück auf das ursprüngliche Verhältnis MenschErde» (IV, S. 820).

Des weiteren, und dies macht die empirische Analyse von K. VORLAUFER 1975 deutlich, braucht es heute wissenschaftliche Anstrengungen, die sich unmittelbar an der Grundeigentumsproblematik orientieren und die der Vielfalt von Besitz-, Eigentums- und Nutzungsformen und ihrer dynamischen Verflechtung im Raum gerecht zu werden 
vermögen. In diesem Sinne hat LUNDEN, wie schon erwähnt, einen empirisch belegten Beitrag zur Landnutzungstheorie geleistet, indem er klarere Vorstellungen über die Faktoren der Landnutzung entwickelt hat, insbesondere über die Aktivitätsstruktur von Eigentümergruppen, die am Raum unterschiedlich interessiert erscheinen.

Am Beispiel der Stadt Basel haben es W.LASCHINGER und L.LÖTSCHER unternommen, Landeigentum und Landnutzung vor allem unter dem dynamischen Aspekt modell-theoretisch zu fassen (Abb.3). Wesentlich an diesem Modell, das 1975 erstmals und 1978 überarbeitet publiziert wurde, ist der hohe Grad an empirischer Erprobung und die daraus abgeleitete Differenzierung der Raumnut-

\section{Soziales System Dynamik}

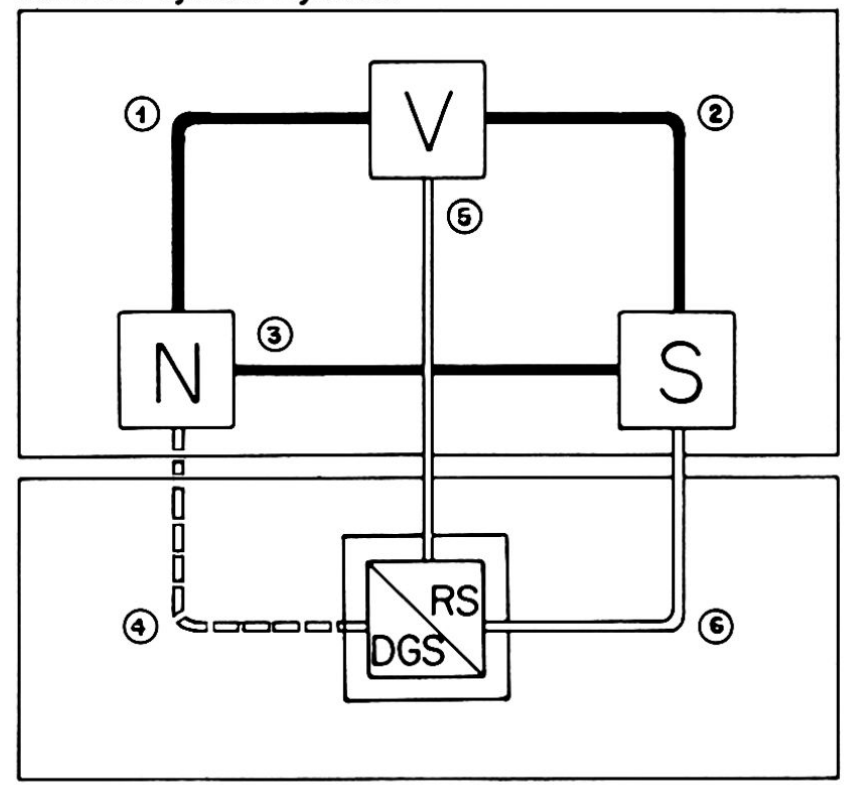

Räumliches System Lebensraum

Abb. 3:

Modell der Lebensraum-Dynamik

(Nach LASCHINGER und LÖTSCHER, 1978)

$N$ - Nutzer

V - Verfüger (Besitzer)

$S$ - Schaffer

DGS - Stelle (Standort) einer Daseinsgrundfunktion

RS - Renditestelle

$1 \quad N-V$

a) Abschluß oder Kündigung eines Mietvertrages

b) Bezahlen einer Miete

2 V-S

a) Abschluß eines Bauvertrages

b) Die $V$ bezahlen Bau- und Unterhaltskosten

$3 \quad \mathrm{~S}-\mathrm{N}$ (wie auch 1 und 2 )

Beeinflussung durch Werbung

$4 \mathrm{~N}$ gebrauchen eine räumliche Einrichtung an einem Standort als DGS

5, 6 V, S gebrauchen eine räumliche Einrichtung an einem Standort als RS zungsdynamik und ihrer Aktivatoren. Die Eigentümer von Land, als "Stellenverfüger» bezeichnet, nehmen danach eine klar definierte Stellung im Raumnutzungsgeschehen ein. Ihre theoretische Einordnung ins Raumsystem auf der Abstraktionsstufe des vorliegenden Modells trägt der realistischen Vielfalt Rechnung und verbindet direkte räumliche Aussagen (über die «Stellen») mit Nutzungsabsichten (Verhalten) und eigentumsrechtlichen Qualitäten der an der Raumdynamik Beteiligten. Ausgehend von den allgemein feststellbaren Funktionsverlagerungen in den westeuropäischen Städten ist somit zumindest ein Entwurf geschaffen worden, welcher die Bedeutung des Grundeigentums für das Raumsystem erkennt und diese Erkenntnis in einem differenzierten Basismodell zur Diskussion stellt.

\section{Grundeigentum zwischen Freiheit und Gebundenheit}

Im Modell von LÖTSCHER und LASCHINGER, wie auch in jenem von CORDEY, wird die Standortsrendite als der wichtigste Antrieb für Grundeigentum, bzw. für die Dynamik des Bodenmarktes bezeichnet. Es ist einleuchtend, daß eine ausschließlich auf Maximierung der Standortsrendite zielende Eigentumsstrategie wohl ökonomisch verständlich, jedoch lebensräumlich folgenschwer sein wird. Schon sind deshalb auch in Ländern der freien Marktwirtschaft rechtliche Korrektursysteme gegen einen exzessiven Bodenmarkt entwickelt worden, und es muß von Fall zu Fall entschieden werden, ob die legalen Lenkungsmittel des Bodenmarktes den Anforderungen einer weitsichtigen Raumplanung zu genügen vermögen. Am Beispiel des großstädtischen Baustrukturwandels in Frankfurt-West kommt E. GIESE 1977 unter anderem zum Schluß, daß die Bodenrente bzw. der Bodenpreis nach wie vor das grundlegende Instrument sei, mit welchem der städtische Umbau vorangetrieben werde, und $\mathrm{da} ß$ die bestehende Bauleitplanung negative Begleiterscheinungen des freien Bodenmarktes (wie Bodenspekulation, Wohnraumvernichtung, Hausbesetzungen) nicht verhindern könne. Es wäre irreführend, diese Einsicht unbesehen auch für andere Räume als gültig zu betrachten, weil die sozialräumlichen Bedingungen vielfach variieren. So kommt W. BAILER 1975 in einer geographischen Untersuchung der Bodenpreise in Furttal (Kanton Zürich) zu einer weiteren Beurteilung der Einflußfaktoren der Landdynamik. Regressionsanalytisch erhält er folgenden Befund: "An erster Stelle steht die Entfernung des Grundstückes von der Stadtgrenze... An zweiter Stelle hat die Art der Bauzone und an dritter Stelle die Entfernung eines Grundstückes vom Dorfzentrum einen Einfluß auf den 
(Boden-) Preis» (a.a. O., S. 109). Obschon die Exposition der Grundstücke im Falle des Furttales keine bestimmende Rolle spielt, sind die lebensräumlichen Lagebedingungen, besonders die Stellung im Zentralitätssystem der Siedlungen, bedeutungsvoll, weil sie die Nutzungsmöglichkeiten des Bodens und damit den Wert des Grundeigentums vorzugsweise bestimmen.

Der Bodenmarkt des Furttales, am dynamischen Stadtrand Zürichs gelegen, verläuft jedoch nicht ausschließlich nach den Gesetzen des Marktes; im Gegenteil hat BAILER einen prägenden Einfluß der kommunalen Bodenpolitik beobachtet. Je nach der gemeindlichen Situation sind mehr oder weniger restriktive Eingriffe auf den Bodenmarkt festzustellen; was «sich bis zu einem gewissen Grad im äußeren Bild einer Gemeinde» auswirkt (a.a.O., S. 117). Wenn im Furttal das bodenpolitische Instrumentarium uneinheitlich und unkoordiniert gehandhabt wird, so widerspiegelt sich darin die gegenwärtige Realität der Schweizer Kulturlandschaft, deren Grundeigentum auch im Zeichen eines tiefgreifenden Landschaftswandels regional recht unterschiedlich betroffen und allgemein sehr zurückhaltend eingeschränkt worden ist.2)

\section{Ausblick: Grundeigentum als räumliche Verantwortung!}

Die beeindruckende Hektik des technologischen Landschaftswandels unserer Tage hat dem menschlichen und damit auch dem wissenschaftlichen Bewußtsein neue Horizonte eröffnet. Für unsere Fragestellung erlangt die Verantwortung des Grundeigentums für den Nutzungswandel ein neues, kritisches Gewicht. Hat sich - ich frage jetzt vor allem für die Schweiz - der freie Eigentümer der Versuchung einer immer höheren Inwertsetzung des Landes gewachsen gezeigt? Ich meine, zahlreiche Beispiele des Mißbrauchs, der Landschaftsverschandelung, ja der Landschaftszerstörung rufen nach Korrekturen eines exzessiven Freiheitsrechtes im Raum, nach einer Harmonisierung des Verhältnisses zwischen dem Eigentum des Einzelnen und der Nutzung durch Viele. Die integrale Einheit des geographischen Raumes verlangt die vielbeschworene ökologische Solidarität zwischen allen menschlichen Partnern eines Lebensraumes, besonders jene zwischen den landbestimmenden Eigentümern und den vielen Nutzern.

Noch hat sich diese Erkenntnis in der schweizerischen Öffentlichkeit nicht voll durchgesetzt, obschon die Volksabstimmung über das Schweizer Raumplanungsgesetz vom 13. Juni 1976 nur ganz knapp (mit $51 \%$ Nein) verworfen worden ist. Mit diesem ersten Raumplanungsgesetz wäre eine maßvolle Barriere gegen eine ungeregelte Vernutzung des Bodens errichtet worden, der sich jeder Grundbesitzer mit Sinn für einen intakten Lebensraum und mit Verantwortung für das Gesamte hätte unterstellen können. Inwieweit die "theoretische» Materie der Raumplanung den negativen Abstimmungsvorgang mitbestimmt hat, bleibe dahingestellt. Als Humangeograph, der sich insbesondere mit dem menschlich organisierten Raum befaßt, müßte man deshalb wohl vermehrt den Zusammenhang zwischen der Regionalplanung (in Zielsetzung, Programm und Sprache) und der betroffenen Regionalbevölkerung beachten.3) Mit dem zweiten Raumplanungsgesetz vom 22. Juni 1979 wird nun ein revidiertes Planungsinstrument zur Geltung gelangen, das aber - von den regionalen Entwicklungstrends her beurteilt - eine zusätzliche Wachsamkeit auf kommunaler und kantonaler Ebene erfordert.

Die Grundbesitzproblematik müßte eigentlich den Geographen in besonderem Maße ansprechen, sie betrifft die Rechtsregeln, nach welchen die Landschaft durch den Menschen beansprucht und gestaltet werden kann. Allein schon durch den Gesetzesanspruch auf Raumplanung ist die absolute Priorität des räumlichen Eigentums bis zu einem gewissen Grade abgeschwächt worden; die Umweltschutzbestrebungen schränken die Eigentumsherrschaft über den Raum weiter ein, so daß heute Politiker und Juristen, wie M. LENDI, vom «geographischen" Ansatz her Grundeigentum und Bodennutzungsrecht neu diskutieren. In diesem Sinn haben H.C.BINSWANGER u.a. 1978 in einem Beitrag zur Totalrevision der Schweizer Bundesverfassung eine Neufassung des Grundeigentums postuliert. Dabei greifen die Autoren auf alteidgenössische Nutzungstraditionen, wie z. B. auf jene der "Allmende», zurück und versuchen, sie für die heutige Lebenssituation neu anzuwenden: "Für eine neue Rechtskonzeption, welche dem Anspruch der Umweltgerechtigkeit genügen will, ist entscheidend, daß die Erhaltung und der Schutz der natürlichen Umwelt zu einem konstituierenden Bestandteil des Eigentums werden. Die Eigentumsrechte sollen nicht mehr primär als umfassend und absolut konstruiert werden, um sie dann nachträglich durch öffentlich-rechtliche Vorschriften zum Schutze der Umwelt wieder einzuschränken. Vielmehr sind die Erfordernisse der Umwelterhaltung als integrierende Bestandteile des Eigentums zu konzipieren. Die Verknüpfung individueller wirtschaftlicher

2) Einschränkende Bundesbeschlüsse waren die "Lex von Moos» (Bundesbeschluß über den Erwerb von Grundstücken durch Personen im Ausland vom 23. März 1961), mit Änderungen 1965 und 1970 sowie die «Lex Furgler» vom 21. März 1973 mit der letzten Änderung vom 24. Juni 1977.

3) Vgl. hiezu die Zürcher Diplomarbeit von BARBARA GAL LUSSER (1979): Regionale Entwicklungskonzepte und ihre Verankerung in der Bevölkerung. Empirisch-methodische Untersuchungen am Beispiel: Oberes Emmental. 
Nutzung des Eigentums und Einhaltung der ökologischen Bedingungen kommt in der Idee des «Patrimoniums» zum Ausdruck. Sie gibt jedem Einzelnen ein - ideelles - Miteigentum an der Umwelt und zugleich entsprechend der heutigen Eigentumsverteilung ein privates Dominium zur wirtschaftlichen Nutzung» (a. a. O., S. 113).

Unter den öffentlichen und gemeinnützigen Grundeigentümern der Schweiz sind beispielsweise die Bürgergemeinden von größerer Wichtigkeit, nicht nur für die Wohlfahrt der Bevölkerung, sondern auch für die Landschaftsgestaltung. Deshalb dürfte sich eine systematische Berücksichtigung der räumlichen Institution «Bürgergemeinde» gerade in geographischen Untersuchungen als recht ergiebig erweisen, bestimmen doch die Bürgergemeinden die innere Struktur, die Dynamik und damit die $\mathrm{Zu}-$ kunft der Kulturlandschaft entscheidend mit. ${ }^{4}$ )

\section{*}

Zusammenfassend läßt sich feststellen, daß die heutige Problematik des Grundeigentums wegen ihren räumlichen Konsequenzen nach einer aktiven Beteiligung des Geographen ruft, wie dies auch J.BASTIÉ 1965 aus französischer Sicht zum Ausdruck gebracht hat: "l'intervention publique, pour être efficace et ne pas aller à l'encontre du but recherché, demande une bien meilleure connaissance des faits qui ne peut résulter que d'études précises et approfondies. Il y a là un vaste champ d'études pour les géographes» (a.a. O., S. 713).

Das Grundeigentum als räumliche Funktion der Kultur wie als Regel-Element der Raumdynamik sollte systematischer in geographische Untersuchungen einbezogen werden. Weil die Grundeigentumsverhältnisse den Lebensraum entscheidend mitbestimmen, ihn verändern und die gesamte $\mathrm{Be}$ völkerung eines Gebietes betreffen, würde ihr Einbezug unsere Arbeit vertiefen und umfassender gestalten. Aus dieser methodischen Erweiterung könnten konstruktive Erkenntnisse erwachsen, die als geographischer Beitrag zur räumlichen Theorie des Grundeigentums auch von allgemeinem Interesse wären.

\section{Literaturverzeichnis}

BAILER, W. (1975): Untersuchung der Bodenpreise im Furttal (Kt.Zürich) unter Berücksichtigung siedlungsgeographischer Faktoren. Diss. Zürich.

BASTIÉ, J. (1965): Le sol, élément primordial du paysage urbain. Annales de Géographie 74, S. 708-713. Paris.

4) Nach K. BUCHMANN 1977 gehören $33 \%$ des Schweizer Waldes Bürgergemeinden und ähnlichen Korporationen; im Kanton St. Gallen besitzen die Bürgergemeinden $11 \%$ des Kulturlandes, $47 \%$ des Waldes und $62 \%$ der Alpen.
BINSWANGER, H. C. u. a. (1978): Eigentum und Eigentumspolitik. Zürich.

BOBEK, H. (1959): Die Hauptstufen der Gesellschafts- und Wirtschaftsentfaltung in geographischer Sicht. Die Erde 90, S. 259-298. Berlin.

BRADLEY, L.F. and BEAULIEU, A. (1973): Social and geographical aspects of agricultural land use in Prince Edward Island. Geograph. Paper 54, Environment Canada Lands Directorate, Ottawa.

BUCHMANN, K. (1977): Die Bürgergemeinde - Idee und Wirklichkeit. St. Gallen.

CADMAN, D. and AUSTIN-CROWE, L. (1978): Property Development. London.

CORDEY, P. (1978): De l'espace produit à l'espace vécu. Geographica Helvetica 33, S. 87-92. Bern.

CRESSWELL, R. (1970): Parenté et propriété foncière dans la montagne libanaise. Études rurales 40 , S. 7-79. Paris.

GALLUSSER, W. A. (1979): Veränderungen im Grundeigentum als Ausdruck der Raumdynamik. In: Siedlungsgeographische Studien. Festschrift für Gabriele Schwarz, S. 465-482. Hrsg. W. Kreisel, W. D. Sick und J. Stadelbauer. Berlin, New York 1979.

GALLUSSER, W. A. (Im Druck): Remarques méthodologiques sur la représentation de la dynamique de l'environnement, basée sur des cartes de la propriété foncière. Caen.

GIESE, E. (1977): Der Einfluß der Bauleitplanung auf die wirtschaftliche Nutzung des Bodens sowie den Boden- und Baumarkt in Großstädten der Bundesrepublik, dargestellt am Beispiel der Frankfurter Innenstadtplanung. Geograph. Zeitschr. 65, S. 109-123. Wiesbaden.

HARTKE, W. (1956): Die «Sozialbranche» als Phänomen der geographischen Differenzierung der Landschaft. Erdkunde, 10, S. 257-269. Bonn.

HASSINGER, H. (1929): Können Kapital, Volksvermögen und Volkseinkommen Gegenstände wirtschaftsgeographischer Betrachtung sein? Geograph. Jahresbericht aus Österreich, 14 und 15. Wien.

JOLLER, R. (1969): Die Wandlungen im Grundeigentum zwischen 1941 und 1965, untersucht an einigen Gemeinden des Kantons Graubünden. Diss. Hochschule St. Gallen. Bern.

LASCHINGER, W. und LÖTSCHER, L. (1978): Basel als urbaner Lebensraum. Basler Beiträge zur Geographie 22-23. Basel.

LENDI, M. (1976): Der Funktionswandel des Eigentums - Rechtl. Aspekte. DISP 40, S. 10-15. Zürich. LUNDEN, TH. (1977): Land use decisions in a timespace framework - some Stockholm examples. Geografiska Annaler 59, Ser. B, S.1-13. Stockholm. 
SCHMIDT, P. H. (1932): Einführung in die allgemeine Geographie der Wirtschaft. Jena

SCHWARZ, G. (1966): Allgemeine Siedlungsgeographie, 3. Aufl. Berlin.

SMITH, D. M. (1977): Human geography. A welfare approach. London.

THOMAS, W.L. (Hg. 1956): Man's role in changing the face of earth. Chicago.

TSCHUDI, A. B. (1968): Land use problems on the urban fringe: the case of Sørkedalen Oslo. Norsk geografisk tidsskrift 22, S. 251-263. Oslo.

TUOR, P. und SCHNYDER, B. (1969): Das schweizerische Zivilgesetzbuch. Eine systematische Darstellung mit Berücksichtigung der Rechtssprechung des schweiz. Bundesgerichts. 8. Aufl. Zürich.

VORLAUFER, K. (1975): Bodeneigentumsverhältnisse und Bodeneigentümergruppen im Cityerweiterungsgebiet Frankfurt/M-Westend. Materialien zur Bodenordnung II. Frankfurter Wirtschafts- und Sozialgeograph. Schriften 18. Frankfurt/M.

WAIBEL, L. (1928): Die Sierra Madre de Chiapas. Verhandlungen und Abhandlungen des 22. Deutschen Geographentages zu Karlsruhe 1927. Breslau.

WILDERMUTH, A. (1970): Marx und die Verwirklichung der Philosophie. Band 2. Den Haag.

\section{Summary}

\section{Geographical aspects of landownership}

Real estate has so far been a very marginal field of research in geography and had been integrated in the study of the cultural landscape; sometimes it was defined as integrated terms like ... «territoriale Herrschaft» or "Betriebsverhältnisse». The modern approach investigating the dynamics of space, seeks to find out the agents responsible for the processes. Thus landownership becomes a methodological tool (this dynamic approach has been discussed in another paper by the same author). Above all regional planning demands a systematic explanation of the relations between real estate and land use. Geographers from Canada, Central Europe and Scandinavia, for example, have already dealt with this aspect. The paper also studies the position of landownership in the spatial system in the light of new models. The statements above point to the landowners' responsibility for land use decisions. In Switzerland, the notion of "property» has of recent become a subject of critical discussion, especially in connection with the revision of the constitution. This discussion is based on the needs of planning, the protection of the environment, and land use justice. As well as the land use policy of larger landowners it can benefit from the scientific experience of geographers who have studied the spatial aspects of real estate. 